\title{
Disturbing findings about the risk of suicide and psychiatric hospitals
}

\author{
Matthew M. Large • Christopher J. Ryan
}

Received: 22 February 2014/Accepted: 26 May 2014/Published online: 11 June 2014

(C) Springer-Verlag Berlin Heidelberg 2014

\section{Introduction}

The results of a study in this issue of the Journal cast further doubt on the appropriateness of suicide risk assessment when patients receive hospital-based psychiatric care. They also raise the disturbing possibility that psychiatric care might, at least in part, cause suicide.

The study, by Hjorth $\varnothing \mathrm{j}$ and associates, is a nested casecontrol study that compared Danish residents who died by suicide between 1996 and 2009 with living age-, sex- and year-matched controls. The authors describe the relationship between suicide and the extent of psychiatric treatment in the previous year [1].

The study found that, compared to those who had no psychiatric treatment in the previous year and after adjustment for other risk factors: those who only received psychiatric medication had 5.8 times the risk of suicide; those with at most outpatient psychiatrist treatment had 8.2 times the risk of suicide; non-admitted patients who had contact with emergency departments had 27.9 times the risk of suicide; and admitted patients had 44.3 times the risk of suicide. Particularly striking are the strength of the associations between emergency room treatment and

M. M. Large ( $\square)$

Department of Mental Health Services, Prince of Wales

Hospital, Randwick, Australia

e-mail: mmbl@bigpond.com

M. M. Large

School of Psychiatry, University of New South Wales, Sydney, Australia

C. J. Ryan

Discipline of Psychiatry and the Centre for Values, Ethics and the Law in Medicine, University of Sydney, Sydney, Australia suicide and between inpatient treatment and suicide. The magnitude of risk ratios of nearly 30 or more for whole groups of patients who have contact with hospital-based services exceed both the risk of suicide associated with major psychiatric disorders [2] and the strength of clinical risk factors for suicide among hospitalized patients [3, 4] by about an order of magnitude.

The strongest associations with suicide estimated by a systematic meta-analysis of controlled studies of inpatient suicide are prior suicide attempts, depressed mood, hopelessness, worthlessness or guilt, and a family history of suicide. However, each of these risk factors confers a risk of inpatient suicide that is about fourfold when compared to other inpatients who do not have these risk factors [3]. The strongest risk factors for suicide after discharge from psychiatric hospitals are prior suicide attempts, and depressive symptoms, which have a strength of association that is below fourfold compared to other discharged patients who do not have these risk factors [4].

\section{Doubt about the appropriateness of suicide risk categorization}

There is a widely held assumption that by combining clinical risk factors, patients can be usefully stratified into risk categories that can guide clinical decisions and that all patients presenting with a psychiatric crisis ought to undergo such suicide risk assessments [5, 6]. However, the Hjorthøj study suggests that the setting in which a suicide risk assessment is conducted is a more powerful indicator of the likelihood of future suicide than any clinical risk factor that the assessment might include. It would seem sensible, for example, all things being equal, to regard a non-depressed person undergoing psychiatric review in the 
emergency department as at far greater risk than a person with depression, who has only ever been treated in the community.

An earlier study by the same authors highlighted the impotence of suicide risk assessment in psychiatric inpatients [7]. That study examined the risk factors for suicide within the population of Danish psychiatric inpatients. It analyzed 279 suicides among 356,712 admissions between 1997 and 2006. During the period of the study one in 1,279 admissions ended in a suicide. The incidence of suicide was 860 per 100,000 inpatient years, a figure 72 times greater than the incidence of suicide in the Danish community $[7,8]$. Of the 356,712 admissions a total of 24,147 (about $7 \%$ of the total) could have been regarded as 'high risk' based on the presence of combined risk factors that included a past history of a suicide attempt, a recent suicide attempt (within the week before psychiatric admission), and a diagnosis of an affective disorder. Of these high-risk admissions, 34 ended in suicide at a rate of one suicide per 710 admissions. The remaining 332,565 admissions could be regarded as low risk because they featured fewer than three risk factors. Seven in eight suicides occurred among low-risk admissions, at a rate of one suicide per 1,357 admissions. Low-risk inpatients had a suicide rate of 800 per 100,000 bed years which is 67 times the Danish national suicide rate.

The modest discriminating power of suicide risk assessments, and the subsequent failure of risk assessment to identify a truly low-risk group or a useful high-risk group is also evident in psychiatric presentations to the emergency department. A recent English study by Steeg and associates [9] examined 18,680 people who were treated in emergency departments for a total of 29,571 episodes of self-harm. In the next 6 months, 92 died by suicide at an annualized rate of 985 per 100,000 patients, which is almost 100 times the English national suicide rate. The authors described a method of identifying those at higher risk of subsequent suicide based on the presence of combined risk factors that included presence of a prior history of self-harm, a more lethal method, homelessness, living alone and a current psychiatric disorder. From the data provided it can be calculated that about 16,600 episodes of self-harm were classified as high risk; 92 of which were followed by suicide within 6 months. Hence, $0.5 \%$ of all high-risk categorizations were true positives and 99.5\% were false positives. While this risk categorization might appear to have some value because if its high sensitivity (89\%), we now know from the Hjorth $\varnothing j$ study that the suicide rate of those who have treatment for psychiatric issues in the emergency department is very high compared to the general population. In the Steeg study about 13,000 people received a low-risk classification after an episode of self-harm. Of these, nine died by suicide. If we assume that there were no repeated episodes of self-harm in this lowrisk group, a conservatively low estimate of the suicide rate for low-risk patients is 138 per 100,000 per annum which is 14 times the English national suicide [10].

In both the English emergency department patients [9] and the Danish study of inpatients [7] only a very small proportion of high-risk patients died by suicide, yet the suicide rate among low-risk patients greatly exceeded that of the general community. We have argued elsewhere that findings like these demonstrate that clinical risk assessment has no utility in directing inpatient clinical decision-making $[11,12]$.

Clinical suicide risk categorization can sort inpatients into those at statistically higher and lower risk for suicide, but both the positive predictive value and specificity of this categorization are too low to be useful. It would only make sense to target a specific suicide lowering intervention to high-risk inpatients if the intervention concerned was effective enough, benign enough and/or cheap enough, to justify its benefits to the very small proportion of high-risk people who will actually suicide; while at the same time being so ineffective, toxic and/or expensive that its use could not be justified in low-risk inpatients who would still have a much higher suicide rate than the general community? Since there is no such effective/ineffective, benign/ toxic and cheap/expensive intervention, suicide risk categorization of those receiving hospital-based treatment for a psychiatric disorder cannot rationally inform management decisions.

\section{Possible implications of a strong and dose-dependent relationship between levels of psychiatric care and suicide}

We have also argued elsewhere that a proportion of the suicides that occur during, or shortly after, psychiatric hospitalization might be properly regarded as "nosocomial" - that is primarily due to factors inherent in hospitalbased care [13]. The Hjorthøj study demonstrated a statistically strong and dose-dependent relationship between the extent of psychiatric treatment and the probability of suicide. This relationship is stepwise, with significant increases in suicide risk occurring with increasing levels of psychiatric treatment. The authors understandably caution that "the association is likely one of selection (rather than causation), in that people with increasing levels of psychiatric contact also are more severely at risk of dying from suicide". This is undoubtedly part of the reason for the association, but it is not possible to be sure that an element of causation may not also be contributing.

Associations that are strong, demonstrate a dose-effect relationship, and have a plausible mechanism are more 
likely to indicate a causal relationship than associations that lack these characteristics [14]. There is now little doubt that suicide is associated with both stigma [15] and trauma [16] in the general community. It is therefore entirely plausible that the stigma and trauma inherent in (particularly involuntary) psychiatric treatment might, in already vulnerable individuals, contribute to some suicides. We believe it is likely that a proportion of people who suicide during or after an admission to hospital do so because of factors inherent in that hospitalization. Such suicides ought to be regarded as nosocomial. Perhaps some aspects of even outpatient psychiatric contact are suicidogenic. These strong stepwise associations urge that we pay closer attention to this troubling possibility.

Conflict of interest Dr. Large and Dr. Ryan have provided expert evidence in civil and coronial matters following the suicide of psychiatric inpatients.

\section{References}

1. Hjorthøj CR, Madsen T, Agerbo E, Nordentoft M (2014) Risk of suicide according to level of psychiatric treatment-a nationwide nested case control study. Soc Psychiatry Psychiatr Epidemiol. doi:10.1007/s00127-014-0860-x

2. Nordentoft M, Mortensen PB, Pedersen CB (2011) Absolute risk of suicide after first hospital contact in mental disorder. Arch Gen Psychiatry 68:1058-1064. doi:10.1001/archgenpsychiatry.2011.113

3. Large M, Smith G, Sharma S, Nielssen O, Singh SP (2011) Systematic review and meta-analysis of the clinical factors associated with the suicide of psychiatric in-patients. Acta Psychiatr Scand 124:18-29. doi:10.1111/j.1600-0447.2010.01672.x

4. Large M, Sharma S, Cannon E, Ryan C, Nielssen O (2011) Risk factors for suicide within a year of discharge from psychiatric hospital: a systematic meta-analysis. Aust N Z J Psychiatry 45:619-628. doi:10.3109/00048674.2011.590465

5. Wortzel HS, Homaifar B, Matarazzo B, Brenner LA (2014) Therapeutic risk management of the suicidal patient: stratifying risk in terms of severity and temporality. J Psychiatr Pract 20:63-67. doi:10.1097/01.pra.0000442940.46328.63

6. Berman AL, Silverman MM (2013) Suicide risk assessment and risk formulation part II: suicide risk formulation and the determination of levels of risk. Suicide Life Threat Behav. doi:10. 1111/sltb.12067 (e-published ahead of print)

7. Madsen T, Agerbo E, Mortensen PB, Nordentoft M (2012) Predictors of psychiatric inpatient suicide: a national prospective register-based study. J Clin Psychiatry 73:144-151. doi:10.4088/ JCP.10m06473

8. Large MM, Nielssen OB (2012) Risk factors for inpatient suicide do not translate into meaningful risk categories-all psychiatric inpatients are high-risk, author reply 1034-5. J Clin Psychiatry 73:1034-1035. doi:10.4088/JCP.12lr07795

9. Steeg S, Kapur N, Webb R, Applegate E, Stewart SL, Hawton K, Bergen H, Waters K, Cooper J (2012) The development of a population-level clinical screening tool for self-harm repetition and suicide: the ReACT self-harm rule. Psychol Med 42:2383-2394. doi:10.1017/S0033291712000347

10. Large M, Ryan CJ (2012) Screening for suicide: a comment on Steeg et al. Psychol Med 42:2011-2012. doi:10.1017/S0033291 712001377

11. Large M, Ryan C, Nielssen O (2011) The validity and utility of risk assessment for inpatient suicide. Australas Psychiatry 19:507-512. doi:10.3109/10398562.2011.610505

12. Ryan C, Nielssen O, Paton M, Large M (2010) Clinical decisions in psychiatry should not be based on risk assessment. Australas Psychiatry 18:398-403. doi:10.3109/10398562.2010.507816

13. Large M, Ryan C, Walsh G, Stein-Parbury J, Patfield M (2014) Nosocomial suicide. Australas Psychiatry 2014(22):118-121. doi:10.1177/1039856213511277

14. Hill AB (1965) The environment and disease: association or causation? Proc R Soc Med 58:295-300

15. Schomerus G, Evans-Lacko S, Rusch N, Mojtabai R, Angermeyer MC, Thornicroft G (2014) Collective levels of stigma and national suicide rates in 25 European countries. Epidemiol Psychiatr Sci. doi:10.1017/S2045796014000109 (e-published ahead of print)

16. Panagioti M, Gooding PA, Tarrier N (2012) A meta-analysis of the association between posttraumatic stress disorder and suicidality: the role of comorbid depression. Compr Psychiatry 53:915-930. doi:10.1016/j.comppsych.2012.02.009 